\title{
SAGA and GAIN for great apes
}

\author{
Tetsuro Matsuzawa ${ }^{1,2}$
}

Published online: 18 December 2015

(C) Japan Monkey Centre and Springer Japan 2015

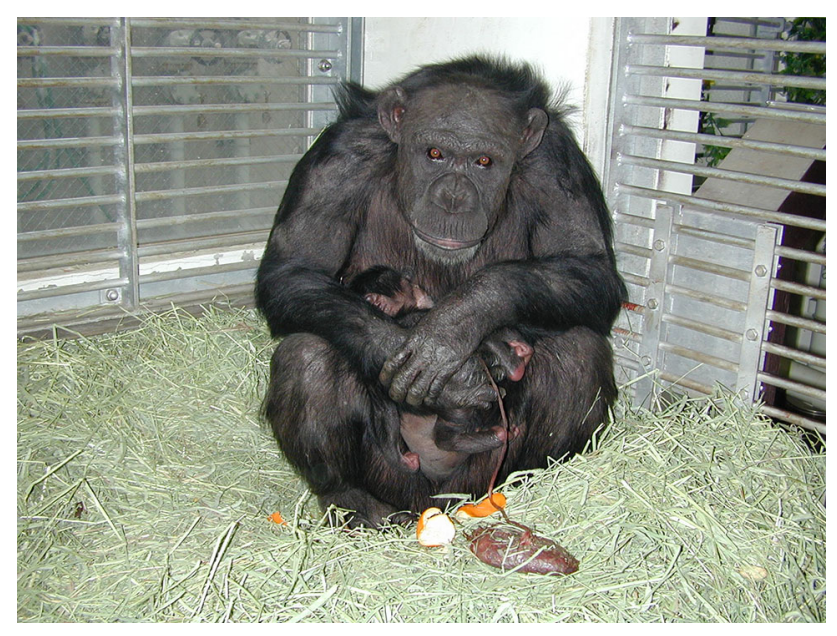

Chimpanzee Ai gave birth to her son, named Ayumu, on April 24th 2000, when she was 24 years old. This year, Ayumu turned 15 and has become the alpha male of the PRI chimpanzee community (Photo credit: Tetsuro Matsuzawa)

Starting from this issue, the front cover of Primates will follow a new convention: the same photograph will appear on all four issues per volume. A new eye-catching cover shot for each new volume. The image featured on this edition shows wild chimpanzees at Bossou, Guinea, a field site at which former editor-in-chief of Primates, Yukimaru Sugiyama and colleagues, including me, have done research. Fieldwork has been carried out at Bossou continuously since 1976, for almost four decades, and at

Tetsuro Matsuzawa

matsuzawa.tetsuro.8w@kyoto-u.ac.jp

1 Primate Research Institute, Kyoto University, Inuyama, Japan

2 Japan Monkey Centre, Inuyama, Japan
Mahale, Tanzania, for even longer. This year, the Mahale Mountains Chimpanzee Research Project (MMCRP) celebrates its 50th anniversary. The study of the chimpanzees of Gombe has reached its 55th anniversary. In this article, I take the opportunity to introduce the situation of captive chimpanzees in Japan and the rest of the world.

How many chimpanzees are there in Japan? The answer is 322 individuals in 50 facilities, as of November 25th 2015. Kyoto University has 57 ex-biomedical chimpanzees housed at Kumamoto Sanctuary, and 13 chimpanzees at the Primate Research Institute. Kyoto University allows only cognitive and behavioral research with chimpanzees that fits within the framework of environmental enrichment for promoting animal welfare in captivity. With the exception of the two Kyoto University facilities, the other 48 facilities housing chimpanzees in Japan are all zoos.

Regarding the other great apes, there are: 6 bonobos housed within one facility, Kumamoto Sanctuary; 24 gorillas in 9 zoos; 48 orangutans in 21 zoos; and 179 gibbons in 43 zoos. This data is regularly updated and accessible to the public in English as well as in Japanese. Please visit the following website maintained by GAIN: Great Ape Information Network. http://www.shigen.nig.ac. jp/gain/.

My other aim in this article is to outline briefly the history of the two key organizations within Japan promoting conservation, welfare, and non-invasive scientific research of great apes: SAGA and GAIN. SAGA stands for 'Support for African/Asian Great Apes' and was founded in 1998 in collaboration between researchers of great apes and members of the public. Please visit the SAGA website: http://www.saga-jp.org/indexe.html.

Japan ratified CITES (the Convention on International Trade in Endangered Species of Wild Fauna and Flora) in 1980. In the 1970s, about 150 chimpanzees were imported 
from Africa to Japan for hepatitis C studies; there were formerly three pharmaceutical companies using chimpanzees for biomedical research. In 1998, SAGA began the fight to prevent the use of chimpanzees in invasive biomedical research. In return, on behalf of laboratory researchers, SAGA promoted the post-mortem utilization of chimpanzees. SAGA also initiated the project to maintain an online database of great apes in Japan: GAIN, introduced above. Since 2002, GAIN has been financially supported by the Japanese government: Ministry of Education, Culture, Sports, Science and Technology in Japan (MEXT).

In 2007 all biomedical research was stopped completely in Japan thanks to extensive support from the public. By the end of 2008, all remaining chimpanzees were relocated to a sanctuary run by Kyoto University, Kumamoto Sanctuary: http://www.wrc.kyoto-u.ac.jp/kumasan/indexE.html.

In Japan, biomedical research on chimpanzees has come to an end. The next outstanding problem to address is the use of chimpanzees by the media and in the entertainment business. I currently take the role of president of the International Primatological Society (IPS). The IPS has established a policy statement opposing the media use of nonhuman primates as performers, photo props or actors, see: http://www.internationalprimatologicalsociety.org/ OppositionToTheUseOfNonhumanPrimatesInTheMedia. cfm.

Humans should not separate infant chimpanzees from their mothers. I have witnessed the birth of seven infant chimpanzees at the Primate Research Institute (PRI), Kyoto University, over the past four decades. Provided that the infant clings to its mother after delivery and that the mother embraces her baby, there should be no fundamental problem with maternal rearing. Across the world, there are still a few cases in which people isolate the infant from its mother, where the sole priority, the justification, is to save the life of the infant chimpanzee. We should not permanently separate infant chimpanzees from their mother for any reason. In cases where the veterinarian judges that temporary separation is essential for medical reasons, the infant should be returned to the mother at the earliest possible opportunity following veterinary treatment.

There are some instances where the mother is unable to rear her infant effectively. In such instances humans may provide help to the mother to nurse and nurture her infant. However, human rearing should not be practiced. If you separate infants from their mothers, they will be deprived of normal social development. They will grow up to become mothers who do not rear their infants properly. We have to put a stop to this negative feedback loop. One should not separate the infant chimpanzee from its mother.

This understanding is gradually permeating the zoo community of Japan. The incidence of human-rearing of great apes has decreased markedly. However, there are still isolated cases where the excuse of saving the infant's life is given, but where the purpose is to use the infant in the media and entertainment industry, for example in television programs. We have to put a stop to this damaging practice.

In the USA, captive chimpanzees are now classified as an endangered species along with their wild counterparts. Moreover, the National Institutes of Health (NIH) recently took the decision to stop financial support for the biomedical study of chimpanzees. Such studies will stop altogether in the USA in the near future. In the USA there is a database, similar to GAIN, created by Project ChimpCARE, a group dedicated to improving the wellbeing of chimpanzees, hosted by Lincoln Park Zoo, Chicago. ChimpCARE currently lists 1720 chimpanzees in the USA, see: http://www.chimpcare.org/map.

The countries that have captive chimpanzees, outside of their native Africa, should work in collaboration to promote the welfare of our evolutionary neighbors.

As a last note in the editorial, I am happy to make an announcement: This year, we will publish a series of articles focusing on two great apes-gorillas and orangutansunder the following special feature titles with guest editors.

Understanding the Variability of Gorilla Social Structure Guest editors: Juichi Yamagiwa and Martha Robbins

Research and Conservation of Orangutans (Pongo sp.) in Malaysia

Guest editors: Misato Hayashi, Tomoko Kanamori, and Noko Kuze

I hope you enjoy the first four articles appearing, in this issue. 